\title{
Noise-tolerant Modular Neural Network System for Classifying ECG Signal
}

\author{
Alberto Ochoa \\ Universidad de Colima, 28400 Colima, Mexico \\ E-mail: aochoa@ucol.mx \\ Luis J. Mena and Vanessa G. Felix \\ Universidad Politecnica de Sinaloa, 82199 Mazatlan, Mexico \\ E-mail: 1mena@upsin.edu.mx, vfelix@upsin.edu.mx \\ Apolinar Gonzalez and Walter Mata \\ Universidad de Colima, 28400 Colima, Mexico \\ E-mail: apogon@ucol.mx,wmata@ucol.mx \\ Gladys E. Maestre \\ University of Texas Rio Grande Valley School of Medicine, Brownsville, TX 78520, USA \\ E-mail: gladys.maestre@utrgv.edu
}

Keywords: electrocardiogram signals, machine learning, classification, neural network

Received: April 18, 2017

\begin{abstract}
Millions of electrocardiograms (ECG) are interpreted every year, requiring specialized training for accurate interpretation. Because automated and accurate classification ECG signals will improve early diagnosis of heart condition, several neural network (NN) approaches have been proposed for classifying ECG signals. Current strategies for a critical step, the preprocessing for noise removal, are still unsatisfactory. We propose a modular NN approach based on artificial noise injection, to improve the generalization capability of the resulting model. The NN classifier initially performed a fairly accurate recognition of four types of cardiac anomalies in simulated ECG signals with minor, moderate, severe, and extreme noise, with an average accuracy of 99.2\%, 95.1\%, 91.4\%, and 85.2\% respectively. Ultimately we discriminated normal and abnormal heartbeat patterns for single lead of raw ECG signals, obtained $95.7 \%$ of overall accuracy and 99.5\% of Precision. Therefore, the propose approach is a useful tool for the detection and diagnosis of cardiac abnormalities.

Povzetek: $V$ članku je opisana metoda modularnih nevronskim mrež za prepoznavanje šumnih ECG signalov.
\end{abstract}

\section{Introduction}

The electrocardiogram (ECG) is a non-invasive clinical test that measures and records electrical changes that take place in the heart when it beats [1]. ECG is vastly used for screening, diagnosis, and monitoring of several heart conditions. Most ECGs are recorded and interpreted by health professionals, few of which have received formal training and proper assessment of competency in recording and interpreting ECGs [2,3], and many selfreported their ECG reading skills as inadequate [4]. Therefore, several automated approaches have been developed to increase efficiency and enhance accuracy in interpreting ECG waveforms [5]. This is the case of classification systems based on artificial neural networks (NN), which have become very popular and most widely employed for successful classification of ECG signals [5] because of their natural ability to deal with incomplete or ambiguous input in pattern recognition tasks [6].

An ECG signal consists mainly of five continuous electromagnetic waves namely, P, Q, R, S, and T (Fig.
1). The amplitude, direction, and duration of the waves, and their morphological aspects are analyzed for specific abnormalities. Other important information includes the peak area, called the QRS complex, the duration of the PR and QT intervals, and the deviation of the PR and ST segments. These characteristics can be contaminated by the physical parameters of electronic and mechanical devices, electrical activity of muscles, degradation of the electrode-skin contact, and other causes [7-9]. Noise corruption can generate similar morphologies to the ECG waveform, reducing the discriminating power of heartbeat patterns, and increasing the rate of false alarms for cardiac monitors [9]. Therefore, a large number of NN approaches for ECG classification have included signal preprocessing for noise reduction, using a wavelet transformer (WT) [6,11-13], nonlinear cubic spline interpolation (CSI) [14], fast Fourier transformation (FFT) [15] or band-pass filters [16-19]. 
Nevertheless, current strategies for this critical step, the preprocessing for noise removal, are still unsatisfactory because clinical interpretation often requires even higher signal quality to detect cardiac disorders [20, 21]. For that reason, NN systems for ECG classification that are robust and efficient, and have greater noise tolerance, are needed. In this paper, we develop and test a noise-tolerant ECG signal classifier based on an NN approach. The method uses a modular $\mathrm{NN}$ architecture to perform initial training and testing on a simulated dataset. Ultimately we discriminated normal and abnormal heartbeat patterns for single lead of real ECG signals.

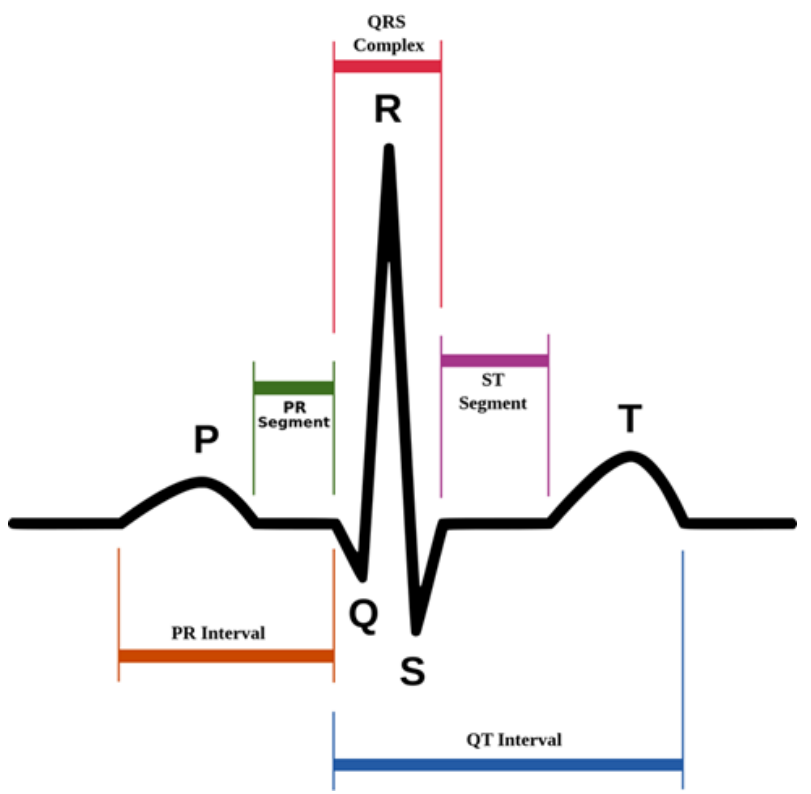

Figure 1: Typical one-cycle ECG signal.

\section{Literature review}

Most NN systems were tested using ECG data from the MIT-BIH arrhythmia database [5], which contains 48 ECG recordings with signals that were band-pass filtered in the frequency range of 0.1 to $100 \mathrm{~Hz}$ and sampled at $360 \mathrm{~Hz}$ [22]. In this sense, Rohan and Patil also used a low pass filter with a cut-off frequency of $30-100 \mathrm{~Hz}$ to pre-process 16 records from the MIT-BIH database [16]. They then employed an NN approach composed by two hidden layers with eight neurons and classified four types of cardiac arrhythmias with an overall accuracy of 99.9\%. Asl et al. used a $5-15 \mathrm{~Hz}$ band-pass filter to remove contamination from 2009 ECG segments, each with 32 RR intervals [17]. The authors developed a three-layered NN with one hidden layer of 20 neurons that classified RR interval signals into four arrhythmia categories with an average accuracy of $99.3 \%$. Das and Ari employed an NN approach with a pre-processing band-pass filter $(3-20 \mathrm{~Hz})$ to reduce noise in 44 records of the database [18]. They classified five types of ECG beats with an S-transform NN approach and achieved 97.9\% of average classification accuracy. In contrast, Tang and Shu eliminated noise from an ECG waveform using high-pass filter with $0.7 \mathrm{~Hz}$ and low-pass filter with
$100 \mathrm{~Hz}$ [19]. Their quantum NN model recognized ECG signals with an overall accuracy of $91.7 \%$.

In two studies the authors used WT technique to remove noise from MIT-BIH records. Javadi et al. proposed a modular NN based on a mixture of experts and negatively correlated learning using stationary WT as a tool for noise reduction [6]. The NN ensemble produced a recognition rate of $96 \%$ for classifying normal heartbeats, premature ventricular contraction arrhythmias and other cardiac abnormalities. Vijayavanan et al. preprocessed ECG signal to remove different kinds of artifacts using discrete WT [12]. They proposed a probabilistic NN approach to discriminate the difference between a normal ECG signal and an arrhythmia affected signal with an accuracy of $96.5 \%$ classification rate. Similarly, Naima and Timemy used discrete WT denoising procedure on ECG data collected from two hospitals in Bagdad [13]. Their discrete WTNN classifier with six neurons in the hidden layer detected acute MI with 95\% accuracy. On the other hand, Güler and Übeyli decomposed ECG signals from the Physiobank database [23] into time-frequency representations also using discrete WT. They classified four types of ECG beats with a total accuracy of $96.9 \%$ through a combined NN model composed by 30 hidden neurons [11]

In its place, Setizwan et al. employed the nonlinear CSI method to estimate and eliminate noise from the baseline ECG of MIT-BIH registers. The implemented fuzzy-neuro learning vector quantization algorithm produced $95.5 \%$ of the overall accuracy rate to classify normal beat and 11 types of arrhythmias [14]. Meanwhile, Vishwa et al. applied direct FFT to remove low frequencies and restore an ECG signal from the MIT-BIH arrhythmia database with the help of inverse FFT [15]. The NN model composed by three and five neurons in first and second hidden layer respectively obtained a detection accuracy of $96.7 \%$.

In contrast, Garg and Sharma used an NN model with two hidden layers to analyze ECG records from the MIT-BIH database with no additional filter and correctly detected normal vs. arrhythmic ECGs with a general accuracy of $96.6 \%$ [24]. Finally, Jadhav et al. used records from the Cardiac Arrhythmia Database of the UCI Machine Learning Repository [25] with no prior filtering for classification of normal and abnormal ECG signals. Their NN approach with two hidden layers resulted in $82.4 \%$ correct classifications [26].

\section{Materials and methods}

The NN classification comprises five stages: (i) simulation of ECG signal, (ii) extraction of features that indicate cardiac abnormalities, (iii) computer generation of normal and abnormal heartbeat patterns, (iv) artificial noise injection, and (v) cardiac rhythm classification on simulated and real ECG signals. 


\subsection{ECG signal simulation}

We used a standard electrocardiographic 12 lead representation of the heart electrical activity [27] divided in the P, PQ, QRS, ST, T and TP sections (Fig. 1), to simulate an ECG signal with specific parameters (Table 1). The resulting signal was composed of different waveforms and frequencies.

\begin{tabular}{lccc}
\hline Section & $\begin{array}{l}\text { Minimum } \\
\text { Voltage }\end{array}$ & $\begin{array}{l}\text { Maximum } \\
\text { voltage }\end{array}$ & Time \\
\hline P & 0 & $0.125 \mathrm{mV}$ & $95 \mathrm{~ms}$ \\
PQ & 0 & 0 & $40 \mathrm{~ms}$ \\
QRS & $-0.1 \mathrm{mV}$ & $0.97 \mathrm{mV}$ & $65 \mathrm{~ms}$ \\
ST & 0 & 0 & $120 \mathrm{~ms}$ \\
T & 0 & $0.16 \mathrm{mV}$ & $180 \mathrm{~ms}$ \\
TP & 0 & 0 & $130 \mathrm{~ms}$ \\
\hline
\end{tabular}

Table 1: Parameters of typical ECG lead.

The $\mathrm{P}$ and $\mathrm{T}$ sections of the simulated ECG signal were similar to waveforms generated by the movement of a piston, which allowed generation of a mathematical model of their behavior [28]. The piston describes an oscillatory motion that can be approximated by a simple harmonic. The position equation of the piston is a function of the angular velocity: $x(t)=r \cdot \cos (\omega t)$, where $x(t)$ is the piston position versus time, $r$ is the radius of the crank and $\omega t$ is the angular velocity of rotation in radians.

The $\mathrm{P}$ wave modeled by the piston motion equation had maximum amplitude of $0.125 \mathrm{mV}$. The $\mathrm{T}$ wave was split into two sections: the first section had maximum amplitude of $0.16 \mathrm{mV}$, a positive slope, and a period of $\mathrm{T}_{1}$; the second section had the same maximum amplitude, a negative slope, and a period, $\mathrm{T}_{2}$, which was less than $T_{1}$. The QRS segment used corresponding voltages at the $\mathrm{Q}, \mathrm{R}$, and $\mathrm{S}$ points and intermediate voltages for the PQ$\mathrm{Q}, \mathrm{Q}-\mathrm{R}, \mathrm{R}-\mathrm{S}$, and S-ST sections. A positive off-set voltage was then added to each value. Each section was further characterized by amplitude and corresponding slope (Table 1). The final output ECG signal, simulated using MATLAB software [29,30], had a duration of 700 $\mathrm{ms}$ for each cycle and was mounted on a signal base of $512 \mathrm{mV}$ amplitude (Fig. 2).

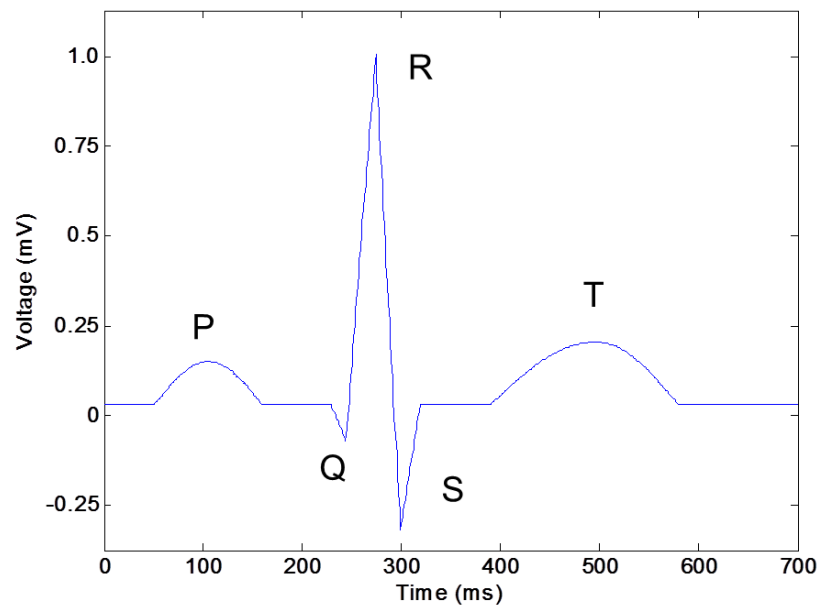

Figure 2: Typical ECG signal simulated in MATLAB.

\subsection{Cardiac abnormalities}

Feature extraction is a key issue in recognition and classification tasks. We used a combination of morphological and timing features to distinguish between a normal heartbeat $(\mathrm{NH})$ and disorders of heart rate and cardiac rhythm. The shape, position, and time duration of $\mathrm{P}, \mathrm{Q}, \mathrm{T}$ waves and the ST segment, were used to identify specific abnormalities (Fig. 3).
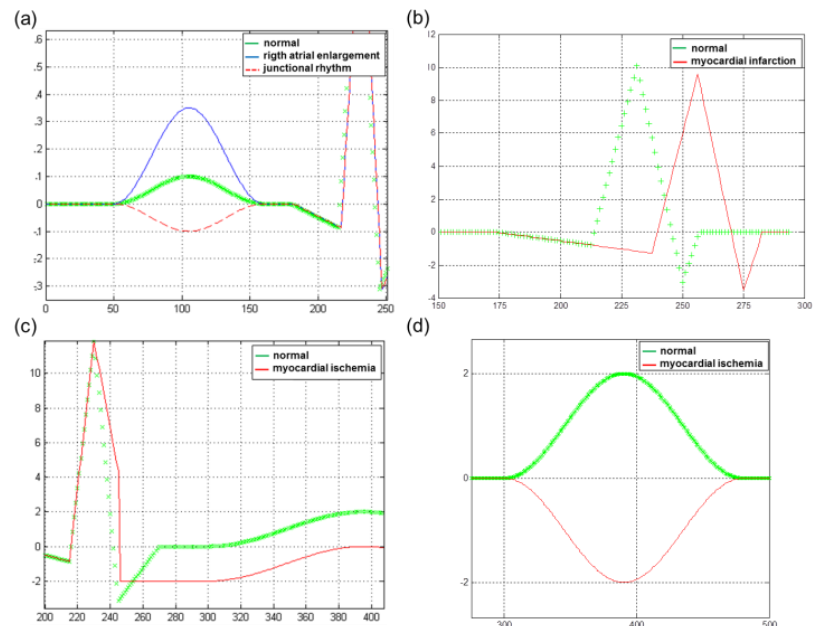

Figure 3: Morphological and timing features of (a) the $\mathrm{P}$ wave; (b) the Q wave; (c) the ST segment; and (d) the T wave, used to detect specific cardiac abnormalities

The $\mathrm{P}$ wave is the first positive deflection on ECG signal, with a normal duration $<120 \mathrm{~ms}$ and amplitude rarely exceeding $0.25 \mathrm{mV}$. Greater amplitude suggests right atrial enlargement (RAE) [31], and an inverted $\mathrm{P}$ wave can indicate junctional rhythm (JR) [32]. The Q wave is the first downward deflection on ECG signal. Pathological Q waves, with a duration $>40 \mathrm{~ms}$ or depth $>0.1 \mathrm{mV}$, can be a sign of current or previous myocardial infarction (MI) [33]. Greater than $0.2 \mathrm{mV}$ depression of the ST segment, which connects the QRS complex and the $\mathrm{T}$ wave, is attributable to cardiac ischemia (CI) [34]. Widespread inversion of the $\mathrm{T}$ wave, the first deflection following the QRS complex, is also associated with CI [35].

\subsection{Dataset}

After extracting the morphological and timing characteristics of the simulated ECG signal, we generated 10000 heartbeat feature vectors (normal and abnormal) for each ECG segment. The dimensions of the feature vectors for the P, Q, ST and T waves were 102, 115,120 , and 200-dimensional respectively. We then built matrices $\left(X_{p}, X_{q}, X_{s t}\right.$ and $\left.X_{t}\right)$ from randomly selected pattern vectors, using 900 for each matrix. These were organized as interspersed normal $\left(x_{N}^{i}\right)$ and abnormal patterns $\left(x_{\mathscr{G}}^{i}\right)$. In the case of a $\mathrm{P}$ wave with two associated pathologies $\left(x_{\mathscr{\alpha}}^{i} x_{b}^{i}\right)$, both abnormal patterns were inserted after each NH pattern:

$$
X_{p}=\left[x_{\mathbb{N}}^{1} x_{\mathbb{L}}^{1} x_{b}^{1} x_{\mathbb{N}}^{2} x_{\mathbb{L}}^{2} x_{b}^{2} \ldots x_{\mathbb{N}}^{n} x_{\mathbb{a}}^{n} x_{b}^{n}\right]
$$

Later, we randomly combined the pattern vectors to generate a total training dataset, composed of 5400 
samples from five classes. The first class was a $\mathrm{NH}$; the other four classes were specific cardiac pathologies for each ECG wave. This takes into account that CI could be attributable to depression of the ST segment or widespread inversion of the $\mathrm{T}$ wave [34]. The total training data was partitioned into two datasets: training and testing set. The testing set was not seen by NN classifier during the training phase. It is only used for testing the generalization of $\mathrm{NN}$ approach after it was trained. We randomly selected the $80 \%$ examples for training, and the rest $20 \%$ examples as testing data.

To assess the robustness of the learned patterns within noisy conditions, and to improve the generalization capability of the resulting NN system, we created artificial corruption in all ECG segments of testing set (Fig. 4) using a Gaussian white-noise model [40]. We injected 1 to $12 \%$ of randomly generated Gaussian white noise [41,42]. We defined quality categories to describe the noise level: minor (1-2\%), moderate $(4-6 \%)$, severe $(8-10 \%)$, and extreme $(12 \%)$. The corrupted testing set was used during the training phase to improve the behavior of NN ensembles while they were trained within noise conditions.

Later we build a corrupted dataset for testing of trained NN with a total of 21600 contaminated ECG segments (17100 NH, $1800 \mathrm{CI}, 900 \mathrm{RAE}, 900 \mathrm{JR}$, and 900 MI segments). In addition, the NN approach was tested on real ECG records of the PhysikalischTechnische Bundesanstalt (PTB) Diagnostic ECG Database [36]. The PTB database contains digitized ECG signals provided by the National Metrology Institute of Germany. This ECG collection included 15 simultaneously measured signals: the conventional 12 leads together with the 3 Frank lead ECGs. Each signal is digitized at 1000 samples per second, with 16 bit resolution over a range of $\pm 16 \mathrm{mV}$ and sampling frequency equal to $1 \mathrm{KHz}$ [36].

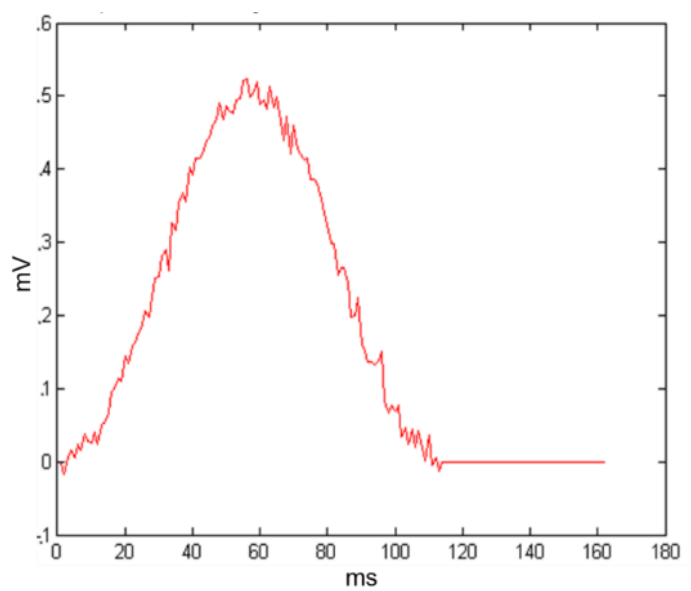

Figure 4: P wave corrupted with 6\% Gaussian white noise.

We selected data from 221 subjects with a clinical summary available, which included ECG records classified as $\mathrm{NH}(\mathrm{n}=52)$ or cardiac abnormalities (148 MI, 14 dysrhythmia and 7 myocardial hypertrophy). For the testing purpose, we considered an unbalanced dataset in favor of arrhythmia data $(76.5 \%)$ to improve the testing generalization capabilities of the NN classifier to recognizing cardiac abnormalities. Lead V1 was chosen for the whole analysis; because it has the largest ratio of atrial to ventricular signal amplitude and therefore can offer more representative characteristics for identifying the common heart diseases [37,38]. The final test set consisted of 884 ECG traces built from 4 heartbeats per individual.

\subsection{Neural network classifier}

The modular NN classifier consisted of four, threelayered, feedforward micro NNs built through Matlab NN toolbox, one for each ECG interval analyzed. A back-propagation algorithm in batch gradient descent with momentum mode [39] and random weights/bias initialization were used for training. Transfer function group was of the hyperbolic tangent-logarithmic sigmoid-linear type for input-hidden-output layers. The learning rate and momentum coefficient were selected as 0.05 and 0.9 respectively. Performance was tested using the mean squared error parameter, computed for differences between the actual outputs and the outputs obtained in each trained micro NN. The training ended, if the total sum of the squared errors was $<0.001$, or when 3000 epochs were reached. The target outputs for $\mathrm{NH}$, RAE, JR, MI, and CI were given by $(0,0,0,0),(0,0,0,1)$, $(0,0,1,0),(0,1,0,0)$, and $(1,0,0,0)$, respectively.

\section{Results}

\subsection{Simulated ECG dataset}

When the NN system was trained using pattern vectors of clean and noised simulated ECG signals, the MSE convergence goal (0.00096) was reached in 109 epochs. The best performance was obtained using 10 (P and $\mathrm{T}$ ) or 5 ( Q and ST) neurons in the hidden layer of the micro NNs. In the first scenario of testing with an artificial corrupted dataset, correct classifications over 10 runs averaged $99.2 \%, 95.1 \%, 91.4 \%$, and $85.2 \%$ for minor, moderate, severe, and extreme noise (Table 2) respectively. Total confusion matrix of each micro NN model for all the levels of noise is shown in Table 3 .

\begin{tabular}{lrrrrrrrrr}
\hline \multirow{2}{*}{ Noise } & \multicolumn{10}{c}{ Micro NN Accuracy (\%) } \\
\cline { 2 - 10 } Level & \multicolumn{1}{c}{ P } & \multicolumn{1}{c}{ Q } & \multicolumn{1}{c}{ ST } & \multicolumn{2}{c}{ T } \\
\cline { 2 - 10 } & NH & RAE & \multicolumn{1}{c}{ JR } & NH & MI & NH & CI & NH & CI \\
\hline Minor & 100 & 99 & 100 & 100 & 100 & 100 & 100 & 97 & 97 \\
Moderate & 97 & 88 & 96 & 100 & 100 & 100 & 100 & 85 & 90 \\
Severe & 96 & 85 & 86 & 100 & 100 & 99 & 100 & 78 & 79 \\
Extreme & 94 & 70 & 74 & 95 & 100 & 96 & 100 & 67 & 71 \\
\hline Average & 96.8 & 85.5 & 89 & 98.8 & 100 & 98.8 & 100 & 81.8 & 84.3 \\
\hline
\end{tabular}

Table 2: Classification performance for contaminated ECG segments.

\begin{tabular}{lcccccccccc}
\hline \multirow{2}{*}{$\begin{array}{l}\text { Estimated } \\
\text { Output }\end{array}$} & \multicolumn{10}{c}{ True output } \\
\cline { 2 - 10 } & NH & RAE & JR & NH & MI & NH & CI & NH & CI \\
\hline \multirow{2}{*}{$\begin{array}{l}\text { NH } \\
\text { Cardiac } \\
\text { anomaly }\end{array}$} & 66184 & 522 & 396 & 67588 & 0 & 67598 & 0 & 55972 & 564 \\
\hline
\end{tabular}

Table 3: Confusion matrix for classification of artificially corrupted ECG segments. 
Overall classification accuracy was $93.9 \%$. The best performance was achieved for the micro NNs, Q and ST, correctly classifying $98.8 \%$ of $\mathrm{NH}$ and $100 \%$ of abnormal segments.

\subsection{Real ECG dataset}

For the last stage of the study the trained NN approach was tested directly on raw ECG traces from PTB database, exclusively for discriminating between $\mathrm{NH}$ and cardiac abnormality. Overall classification accuracy was 95.7\%. The results are shown by a confusion matrix, where each cell contains the number of ECG traces classified for the corresponding combination of estimated and true outputs (Table 4).

\begin{tabular}{lcc}
\hline \multirow{2}{*}{$\begin{array}{l}\text { Estimated } \\
\text { output }\end{array}$} & \multicolumn{2}{c}{ True output } \\
\cline { 2 - 3 } & $\mathrm{NH}$ & Cardiac abnormality \\
\hline $\begin{array}{l}\text { NH } \\
\begin{array}{l}\text { Cardiac } \\
\text { abnormality }\end{array}\end{array}$ & 205 & 35 \\
\hline
\end{tabular}

Table 4: Confusion matrix for classification of real ECG signals.

The total test performance of the NN classifier is displayed in Table 5. This was determined by the computation of evaluation metrics such as Specificity (number of correctly classified $\mathrm{NH}$ over total number of $\mathrm{NH}$ ), Sensitivity (number of correctly classified cardiac abnormalities over total number of cardiac abnormalities), and Precision (number of correctly classified cardiac abnormalities over total number of estimated cardiac abnormalities).

\begin{tabular}{lc}
\hline Evaluation metrics & Values (\%) \\
\hline Specificity & 98.6 \\
Sensitivity & 94.8 \\
Precision & 99.5 \\
Overall classification accuracy & 95.7 \\
\hline
\end{tabular}

Table 5: Total test performance of the NN classifier.

In Table 6 the overall performance of our proposed $\mathrm{NN}$ classifier is compared with the recognition rate of previous NN approaches for EGC classification found in the literature.

\section{Discussion}

Overall, results of the previous studies make it clear that suppression of noise corruption embedded in analysed signals improves the accuracy of NN classifiers. However, filtering parameters, particularly cut-off frequencies and phase response characteristics, should be chosen such that clinical information in ECG signals remains undistorted, while as much noise as possible is removed [43]. This is difficult because the signal and noise often share the same frequencies. Furthermore, adaptive filters do not normally have a sharp delineation between the pass-bands and cut-bands, but rather a slow transition in the filter response. If the clinical signals and noise are close, it may not be possible to remove the noise without removing some of the clinical signal [44].

\begin{tabular}{lcc}
\hline NN approach & $\begin{array}{c}\text { Filtering } \\
\text { technique }\end{array}$ & $\begin{array}{c}\text { Accuracy } \\
(\%)\end{array}$ \\
\hline *Rohan et al. [16] & $30-100 \mathrm{~Hz}$ & 99.9 \\
*Asl et al. [17] & $5-15 \mathrm{~Hz}$ & 99.3 \\
*Das et al. [18] & $3-20 \mathrm{~Hz}$ & 97.9 \\
Güler et al. [11] & WT & 96.9 \\
*Vishwa [15] & FFT & 96.7 \\
*Garg et al. [23] & Non & 96.6 \\
*Vijayavanan [12] & WT & 96.5 \\
*Javadi et al. [6] & WT & 96.0 \\
*Setiawan et al. [14] & CSI & 95.5 \\
Naima et al. [13] & WT & 95.0 \\
*Tang et al. [19] & 0.7-100 Hz & 91.7 \\
Jadhav et al. [26] & Non & 82.3 \\
Method proposed & Non & $\mathbf{9 5 . 7}$ \\
\hline * ECG signals that & were & previously band-pass \\
filtered in the frequency range of 0.1 to 100 Hz and \\
sampled at 360 Hz.
\end{tabular}

Table 6: Comparison of the overall classification accuracy of the proposed method and previous $\mathrm{NN}$ approaches in literature.

Due to filtration problems, several previous studies found that adaptive filtering affected the estimation of morphological parameters in ECG signals [45-47], resulting in changes that simulated CI [48-53]. A reduction in the number of peaks and valleys was particularly misleading [54].

Converse to previous NN approaches for classification of ECG signals, the system proposed trains with clean and noisy data [55]. By using inputs corrupted with randomly sampled noises and various signal-tonoise ratios, we were able to build a robust classifier without an adaptive filter, because the injected noise improves the generalization capability of the NN model [56]. The rationale of this approach is that the perturbation introduced in training by the injected noise can be learned by the NN structure and recognized in the test phase. More exactly, noise injection during the training favors an optimal solution at which the objective function is less sensitive to the change of the input [57]. On the other hand, this approach is based on the premise that an NN method, which can provide accurate classification with noise, is preferable to methods that modify the original signal. Furthermore the modular design based in micro NNs provides a more specific classification for each considered kind of cardiac abnormality. In this sense, the analysis and experiments suggest that by injecting a minor to extreme level of noise in training of $\mathrm{NN}$, the noise patterns can be effectively learned, and the generalization capability of the micro NNs can be improved. Both of these advantages result in substantial performance improvement of $\mathrm{NN}$ for ECG classification in noise conditions, without the inclusion of adaptive filters.

However, although the average classification accuracy and precision of our NN system is competitive, 
the system was tested for the detection of only four types of cardiac pathologies. On the other hand, the first results of the trained NN approach were achieved with artificially generated random Gaussian white noise, without any specific assumption on the origin of the noise. Moreover, the additive noise model can differ to some extent from real ECG records that are corrupted by physiological noise and exhibiting spatial correlation across the individual ECG signals [16]. These limitations require additional research for situations where the nature of the contaminating noises are better known, and the additive artificial noise may be selected according to the particular situation. Therefore, our system requires further verification including information about the noise sources using actual ECG data and classifying other specific types of cardiac disorders.

\section{Conclusion}

We developed a robust and fairly accurate, noise-tolerant NN approach for detecting and diagnosing specific cardiac abnormalities. The modular NN system discriminates between simulated normal and abnormal cardiac rhythms with high accuracy for ECG signals with minor to moderate noise and good accuracy for signals with severe to extreme noise. The previous artificial noise injection stage enables the trained NN classifier to handle noise and recognize cardiac abnormalities on raw ECG signals with high Precision. With further verification, this system could facilitate the use of NN approaches to support clinical decisions.

\section{Acknowledgments}

This paper was supported by the Secretaria de Educación Pública, México (DSA/103.5/15/11115). The authors acknowledge the support of the Programa Interinstitucional para el Fortalecimiento de la Investigación y el Posgrado del Pacífico, which promotes the formation and consolidation of research networks integrated by researchers from universities, technological institutes, and state councils of science and technology of Mexico. GE Maestre is supported partially by the National Institutes of Health and the National Institute of Aging (5 R01 AG036469-05, 1R03AG054186). We acknowledge Sarah Kaip for her editing of English language.

\section{Conflicts of interest}

None declared.

\section{References}

[1] Hurxthal L.M. Clinical interpretation of the electrocardiogram. The New England Journal of Medicine, 1934, Vol. 211, No. 10, pp. 431-437. https://doi.org/10.1056/NEJM193409062111001

[2] Wolff A.R., Long S., McComb J.M., Richley D., Mercer P. The gap between training and provision: a primary-care based ECG survey in North-East
England. The British Journal of Cardiology, 2012, Vol. 19, No. 1, pp. 38-40.

https://doi.org/10.5837/bjc.2012.008

[3] Richley D. New training and qualifications in electrocardiography. British Journal of Cardiac Nursing, 2013, Vol. 8, No. 1, pp. 38-42.

https://doi.org/10.12968/bjca.2013.8.1.38

[4] Som S. Nurse practitioners (and other physician extenders) are not an appropriate replacement for expert physician electrocardiogram readers in routine clinical practice. Journal of the American College of Cardiology, 2015, Vol. 65, No. 1, pp. 106-107. https://doi.org/10.1016/j.jacc.2014.09.075

[5] Jambukia S.H., Dabhi V.K., Prajapati, H.B. Classification of ECG signals using machine learning techniques: A survey. Proc. of IEEE International Conference on Advances in Computer Engineering and Applications, 2015, pp. 714-721. https://doi.org/10.1109/ICACEA.2015.7164783

[6] Javadi M., Arani S.A.A.A, Sajedin A., Ebrahimpour R. Classification of ECG arrhythmia by a modular neural network based on mixture of experts and negatively correlated learning. Biomedical Signal Processing and Control, 2013, Vol. 8, No. 3, pp. 289-296. https://doi.org/10.1016/j.bspc.2012.10.005

[7] Zadeh A.E., Khazaee A., Ranaee V. Classification of the electrocardiogram signals using supervised classifiers and efficient features. Computer Methods and Programs in Biomedicine, 2010, Vol. 99, No. 2, pp. 179-194. https://doi.org/10.1016/j.cmpb.2010.04.013

[8] Micó P., Mora M., Cuesta-Frau D., Aboy M. Automatic segmentation of long-term ECG signals corrupted with broadband noise based on sample entropy. Computer Methods and Programs in Biomedicine, 2010, Vol. 98, No. 2, pp. 118-129. https://doi.org/10.1016/j.cmpb.2009.08.010

[9] Choudhary M., Narwaria R.P. Suppression of noise in ECG signal using low pass IIR filters. International Journal of Electronics and Computer Science Engineering, 2012, Vol. 1, No. 4, pp. 2238 2243.

[10] Li Q., Clifford G.D. Signal quality and data fusion for false alarm reduction in the intensive care unit. Journal of Electrocardiology, 2012, Vol. 45, No. 6, pp. 596-603. https://doi.org/10.1016/j.jelectrocard.2012.07.015

[11] Güler, İ., Übeyli, E.D. (2005). ECG beat classifier designed by combined neural network model. Pattern recognition, 2005, Vol. 38, No. 2, pp. 199 208. https://doi.org/10.1016/j.patcog.2004.06.009

[12] Vijayavanan M., Rathikarani V., Dhanalakshmi P. Automatic classification of ECG signal for heart disease diagnosis using morphological features. International Journal of Computer Science \& Engineering Technology, 2014, Vol. 5, No. 4, pp. 449-455.

[13] Naima, F.A, Timemy A.A. Neural network based classification of myocardial infarction: a comparative study of Wavelet and Fourier 
transforms. In Pattern Recognition, 2009, pp. 337351. INTECH. https://doi.org/10.5772/7533

[14] Setiawan I.M.A., Imah, E.M., Jatmiko, W. Arrhytmia classification using fuzzy-neuro generalized learning vector quantization. Proc. of IEEE International Conference on Advanced Computer Science and Information System, 2011, pp. 385-390.

[15] Vishwa A.M.K., Lal S.D., Vardwaj P. Clasification of arrhythmic ECG data using machine learning techniques. International Journal of Interactive Multimedia and Artificial Intelligence, 2011, Vol. 1, No. 4 pp. 67-70. https://doi.org/10.9781/ijimai.2011.1411

[16] Rohan M.D., Patil A.J. Layered approach for ECG beat classification utilizing neural network. Bioinformatics, 2012, Vol. 2, No. 6, pp. 1495-1500.

[17] Asl B.M., Sharafat A.R., Setarehdan S.K. An adaptive backpropagation neural network for arrhythmia classification using RR interval signal. Neural Network World, 2012, Vol. 6, No. 12, pp. 535-54.

[18] Das M.K., Ari S. ECG arrhythmia recognition using artificial neural network with S-transform based effective features. Proc. of IEEE India Conference Annual, 2013, pp. 1-6. https://doi.org/10.1109/INDCON.2013.6726153

[19] Tang X., Shu L. Classification of electrocardiogram signals with RS and quantum neural networks. International Journal of Multimedia and Ubiquitous Engineering, 2014, Vol 9, No. 2, pp. 363-372.https://doi.org/10.14257/ijmue.2014.9.2.37

[20] Li Q., Rajagopalan C., Clifford G.D. A machine learning approach to multi-level ECG signal quality classification. Computer Methods and Programs in Biomedicine, 2014, Vol. 117, No. 3, pp. 435-447. https://doi.org/10.1016/j.cmpb.2014.09.002

[21] Redmond S.J., Xie Y., Chang D., Basilakis J., Lovell N.H. Electrocardiogram signal quality measures for unsupervised telehealth environments. Physiological Measurement, 2012, Vol. 33, No. 9, pp. 1517-1533. https://doi.org/10.1088/0967-3334/33/9/1517

[22] Moody G.B., Mark R.G. The impact of the MIT$\mathrm{BIH}$ arrhythmia database. IEEE Engineering in Medicine and Biology Magazine, 2001, Vol. 20, No. 3, pp. 45-50. https://doi.org/10.1109/51.932724

[23] Goldberger A.L., Amaral L.A.N., Glass L., Hausdorff J.M., Ivanov P.Ch., Mark R.G., Mietus J.E., Moody G.B., Peng, C.K., Stanley H.E. Physiobank, Physiotoolkit, and Physionet. Components of a new research resource for complex physiologic signals. Circulation, 2000, Vol. 101, No. 23, pp. e215-e220. https://doi.org/10.1161/01.CIR.101.23.e215

[24] Garg P., Sharma A. Detection of normal ECG and arrhythmia using artificial neural network system. International Journal of Engineering Research and Science \& Technology, 2015, Vol. 4, No. 1, pp. 113.
[25] Moses D. A survey of data mining algorithms used in cardiovascular disease diagnosis from multi-lead ECG data. Kuwait Journal of Science, 2015, Vol. 42, No. 2, pp. 206-235.

[26] Jadhav S., Nalbalwar S., Ghatol A. Performance evaluation of generalized feedforward neural network based ECG arrhythmia classifier. International Journal of Computer Science Issues, 2012, Vol. 9, No. 4, pp. 379-384

[27] Ambrose M. ECG. Interpretación clínica. Manual Moderno, Buenos Aires, 2008.

[28] Chierici F., Pignagnoli L., Embriaco D. Modeling of the hydroacoustic signal and tsunami wave generated by seafloor motion including a porous seabed. Journal of Geophysical Research, 2010. Vol. 115, No. C3, pp. 1-15. https://doi.org/10.1029/2009JC005522

[29] Islam M.K., Haque A.N., Tangim G., Ahammad T., Khondokar M.R.H. Study and analysis of ECG signal using MATLAB \& LABVIEW as effective tools. International Journal of Computer and Electric Engineering, 2012, Vol. 4, No. 3, pp. 404408. https://doi.org/10.7763/IJCEE.2012.V4.522

[30] Guda M., Gasser S., El Mahallawy M.S. MATLAB simulation comparison for different adaptive noise cancelation algorithms. Proc. of the International Conference on Digital Information, Networking, and Wireless Communications, 2014, pp. 68-73.

[31] Bille K., Figueiras D., Schamasch P., Kappenberger L., Brenner J.I., Meijboom F.J., Meijboom E.J. Sudden cardiac death in athletes: the Lausanne Recommendations. European Journal of Cardiovascular Prevention \& Rehabilitation, 2006, Vol. 13, No. 6, pp. 859-875. https://doi.org/ 10.1097/01.hjr.0000238397.50341.4a

[32] Cools E., Missant C. Junctional ectopic tachycardia after congenital heart surgery. Acta Anaesthesiologica Belgica, 2014, Vol. 65, No. 1, pp. 1-8.

[33] Das M.K., Khan B., Jacob S., Kumar A., Mahenthiran J. Significance of a fragmented QRS complex versus a $\mathrm{Q}$ wave in patients with coronary artery disease. Circulation, 2006, Vol. 113, No. 21, pp. 2495-2501. https://doi.org/10.1161/ CIRCULATIONAHA.105.595892

[34] Valo M., Moller A., Teupe C. Markers of myocardial ischemia in patients with diabetes mellitus and severe obstructive sleep apnea impact of continuous positive airway pressure therapy. Journal of Diabetes \& Metabolism, 2015, Vol. 6, No. 492, pp. 2-5.

[35] Bakhoya V.N., Kurl S., Laukkanen J.A. T-wave inversion on electrocardiogram is related to the risk of acute coronary syndrome in the general population. European Journal of Preventive Cardiology, 2014, Vol. 21, No. 4, pp., 500-506.

[36] Bousseljot R., Kreiseler D., Schnabel A. Nutzung der EKG-Signaldatenbank CARDIODAT der PTB über das Internet. Biomedizinische Technik/Biomedical Engineering, 1995, Vol. 40, No. s1, pp. 317-318. 
[37] Mainardi L., Sornmo L., Cerutti S. Understanding atrial fibrillation: the signal processing contribution. Morgan \& Claypool Publishers, San Rafael, 2008.

[38] Ramli A.B., Ahmad P.A. Correlation analysis for abnormal ECG signal features extraction. Proc. of 4th National Conference on Telecommunication Technology, 2003, pp. 232-237. https://doi.org/10.1109/NCTT.2003.1188342

[39] Paulin F., Santhakumaran, A. Classification of breast cancer by comparing back propagation training algorithms. International Journal on Computer Science and Engineering, 2011, Vol. 3, No. 1, pp. 327-332.

[40] Szabó B.T., van der Vaart A.W., van Zanten J.H. Empirical Bayes scaling of Gaussian priors in the white noise model. Electronic Journal of Statistics, 2013, Vol. 7, pp. 991-1018. https://doi.org/10.1214/13-EJS798

[41] Grandvalet Y., Canu S. Comments on noise injection into inputs in back propagation learning. IEEE Transactions Systems, Man and Cybernetics, 1995, Vol. 25, No. 4, pp. 678-681. https://doi.org/10.1109/21.370200

[42] An G. The effects of adding noise during backpropagation training on a generalization performance. Neural Computation, 1996, Vol. 8, No. 3, pp. 643-674. https://doi.org/10.1162/neco.1996.8.3.643

[43] Kumar R., Gupta R., Jyoti K., Ranjan A.K. AT89C51 microcontroller based medical two channel ECG module and body temperature measurement with graphics LCD. International Journal of Advanced Research in Computer Science and Software Engineering, 2015, Vol. 5, No. 5, pp. 1321-1326.

[44] Longini R.L., Giolma J.P., Wall III C., Quick R.F. Filtering without phase shift. IEEE Transactions on Biomedical Engineering, 1975, Vol. BME-22, No. 5, pp. 432-433. https://doi.org/10.1109/TBME.1975.324517

[45] Valverde E.R., Quinteiro R.A., Bertran G.C., Arini P.D., Glenny P., Biagetti M.O. Influence of filtering techniques on the time domain analysis of signalaveraged P wave electrocardiogram. Journal of Cardiovascular Electrophysiology, 1998, Vol. 9, No. 3, pp. 253-260. https://doi.org/10.1111/j.1540-8167.1998.tb00910.x

[46] Łęski J.M., Henzel N. ECG baseline wander and powerline interference reduction using nonlinear filter bank. Signal Processing, 2005, Vol. 85, No. 4, pp. 781-793. https://doi.org/10.1016/j.sigpro.2004.12.001

[47] Gregg R.E., Zhou S.H., Lindauer J.M., Helfenbein E.D., Giuliano K.K. What is inside the electrocardiograph?. Journal of Electrocardiology, 2008, Vol. 41, No. 1, pp. 8-14. https://doi.org/10.1016/j.jelectrocard.2007.08.059

[48] Berson A.S., Pipberger H.V. The low-frequency response of electrocardiographs, a frequent source of recording errors. American Heart Journal, 1966, Vol. 71, No. 6, pp. 779-789.

https://doi.org/10.1016/0002-8703(66)90599-0

[49] Bragg-Remschel D.A., Anderson C.M., Winkle R.A. Frequency response characteristics of ambulatory ECG monitoring systems and their implications for ST segment analysis. American Heart Journal, 1982, Vol. 103, No. 1, pp. 20-31. https://doi.org/10.1016/0002-8703(82)90524-5

[50] Tayler D., Vincent R. Signal distortion in the electrocardiogram due to inadequate phase response. IEEE Transactions on Biomedical Engineering, 1983, Vol. BME-30, No. 6, pp. 352356.

https://doi.org/10.1109/TBME.1983.325137

[51] Tayler D.I, Vincent R. Artefactual ST segment abnormalities due to electrocardiograph design. British Heart Journal, 1985, Vol. 54, No. 2, pp. 121-128. https://doi.org/10.1136/hrt.54.2.121

[52] Burri H., Sunthorn H., Shah D. Simulation of anteroseptal myocardial infarction by electrocardiographic filters. Journal of Electrocardiology, 2006, Vol. 39, No. 3, pp. 253258.

https://doi.org/10.1016/j.jelectrocard.2005.11.001

[53] Censi F., Calcagnini G., Triventi M., Mattei E., Bartolini P., Corazza I., Boriani G. Effect of highpass filtering on ECG signal on the analysis of patients prone to atrial fibrillation. Annali dell'Istituto Superiore di Sanità, 2009, Vol. 45, No. 4, pp. 427-431. https://doi.org/10.1590/S0021-25712009000400012

[54] Buendía-Fuentes F., Arnau-Vives M.A., ArnauVives A., Jiménez-Jiménez Y., Rueda-Soriano J., Zorio-Grima E., Osa-Sáez A., Martínez-Dolz L.V., Almenar-Bonet L., Palencia-Pérez M.A. Highbandpass filters in electrocardiography: source of error in the interpretation of the ST segment. International Scholarly Research Notices Cardiology, 2012, Vol. 2012, pp. 1-10. https://doi.org/10.5402/2012/706217

[55] Li B., Tsao Y., Sim K.C. An investigation of spectral restoration algorithms for deep neural networks based noise robust speech recognition. Proc. of 14th Annual Conference of the International Speech Communication Association, 2013, pp. 3002-3006.

[56] Yin S., Liu C., Zhang Z., Lin Y., Wang D., Tejedor J., Fang-Zhen T., Li Y. Noisy training for deep neural networks in speech recognition. EURASIP Journal on Audio, Speech, and Music Processing, 2015, Vol. 2015, No. 1, pp. 1-14. https://doi.org/10.1186/s13636-014-0047-0

[57] Grandvalet Y., Canu S Comments on "Noise injection into inputs in back propagation learning". IEEE Transactions on Systems, Man, and Cybernetics, 1995, Vol. 25, No. 4, pp. 678-681. https://doi.org/10.1109/21.370200 